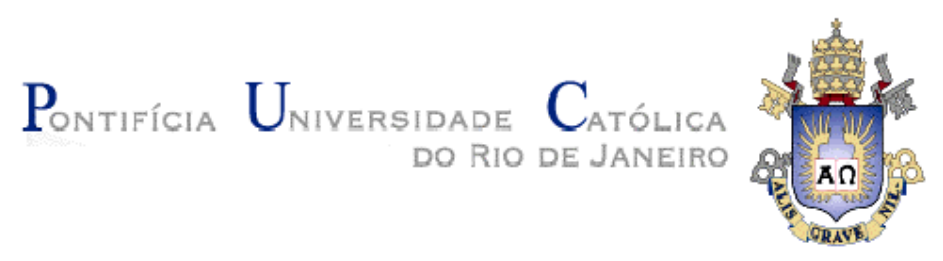

Ana Lucia Vaz da Silva

\title{
NÚMEROS REAIS NO ENSINO MÉDIO Identificando e possibilitando imagens conceituais
}

\section{Tese de Doutorado}

Tese apresentada ao Programa de PósGraduação em Educação do Departamento de Educação da PUC-Rio como parte dos requisitos parciais para obtenção do título de Doutor em Educação.

Orientador: Prof ${ }^{\mathrm{a}}$ Isabel Alice O.M. Lelis

Co-orientadora: Mônica Rabello de Castro

Rio de Janeiro

Março de 2011 

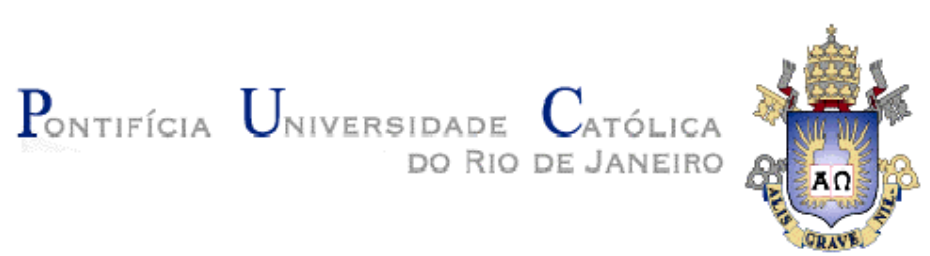

Ana Lucia Vaz da Silva

\title{
NÚMEROS REAIS NO ENSINO MÉDIO Identificando e possibilitando imagens conceituais
}

\begin{abstract}
Tese apresentada como requisito parcial para obtenção do título de Doutor pelo Programa de Pós-Graduação em Educação do Departamento de Educação do Centro de Teologia e Ciências Humanas da PUC-Rio. Aprovada pela Comissão Examinadora abaixo assinada.
\end{abstract}

Prof $^{\mathrm{a}}$ Isabel Alice O. M. Lelis Orientadora Departamento de Educação - PUC-Rio

Profa Mônica Rabello de Castro Co-orientadora UNESA

Profa. Cynthia Paes de Carvalho Departamento de Educação - PUC-Rio

Profa. Hedy Silva Ramos de Vasconcellos

Departamento de Educação - PUC-Rio

Prof. Marcelo Almeida Bairral UFRRJ

Prof. Wanderley Resende UFF

Profa . Denise Berruezo Portinari Coordenadora Setorial do Centro de

Teologia e Ciências Humanas

Rio de Janeiro, 25 de março de 2011. 
Todos os direitos reservados. É proibida a reprodução total ou parcial do trabalho sem autorização da universidade, da autora e do orientador.

\section{Ana Lucia Vaz da Silva}

Graduou-se e licenciou-se em Matemática pela U.F.F. em 1989. É professora de Matemática do Colégio Pedro II desde 1992. Elaborou material didático de Matemática para o Ensino Médio. Possui Mestrado em Educação Matemática na U.S.U.

Ficha Catalográfica


possibilitando imagens conceituais / Ana Lúcia Vaz da Silva; orientadora: Isabel Alice Oswald Monteiro Lelis; coorientadora: Monica Rabello de Castro. - 2011.

$333 \mathrm{f}$. : il. ; $30 \mathrm{~cm}$

Tese (doutorado)-Pontifícia Universidade Católica do Rio de Janeiro, Departamento de Educação, 2011.

Inclui bibliografia

1. Educação - Teses. 2. Número real. 3. Imagem conceitual. 4. Currículo escolar. 5. Ensino médio. 6. Método clínico. I. Lelis, Isabel Alice Oswald Monteiro. II. Castro, Monica Rabello de. III. Pontifícia Universidade Católica do Rio de Janeiro. Departamento de Educação. IV. Título.

CDD: 370 
Dedico este estudo ao Colégio Pedro II, representando a escola pública brasileira, a Professora Doutora Mônica Rabello de Castro representando todos os bons professores deste país 


\section{Agradecimentos}

Agradeço ao meu marido, Mario, pelo carinho, pela força e pela dedicação.

Aos meus filhos, Bruna, Guilherme, Beatriz e Arthur, pelo amor, pela felicidade, renovação e estrutura na/da minha vida.

À meus pais, Fernando e Cândida, e ao meu irmão Marcelo (todos in memoriam), por parte do que sou e tudo que aprendi com eles.

Ao meu irmão, André, pela presença real e constante em meus pensamentos.

À grande amiga, Rosana de Oliveira pela força, alegria e constante ajuda neste trabalho.

Às grandes amigas, Rosaninha, Andreia e Ana Patrícia, pela força incondicional e pelo carinho necessário em momentos difíceis.

Aos diferentes amigos pela força, perseverança e confiança.

A tia Graça e aos meus familiares pelo carinho e preocupação.

Às amigas, presentes do curso de doutorado, Eloiza, Solange, Cristina, Marcela, e Lobélia, pelas diferenças e semelhanças, que nos tornam amigas para sempre.

À Luciana e a Maria Helena pela paz, cuidado e dedicação com meus filhos.

À minha eterna orientadora, Mônica de Castro Rabello, pela coragem e profissionalismo.

Às professoras doutoras Sonia Kramer e Zaia Brandão pelos diferentes exemplos de educadoras.

Aos professores da PUC pelas contribuições dadas ao longo do curso de doutorado. 
À professora doutora Hedy Silva Ramos de Vasconcelos pela contribuição e pela solidariedade dedicadas em momentos difíceis.

Á coordenação do Departamento de Educação da PUC Rio por me ouvir nos momentos difíceis.

À Direção e aos professores da Unidade Centro do Colégio Pedro II, pela colaboração e solidariedade em vários momentos.

Aos alunos Araão, Anselmo, Bernardo, Camila, Clarisse, Carlos, Débora, Emanuel, Felipe, Ingra, Thaís e Vítor pela disponibilidade, pela ajuda, pelo aprendizado e pelas grandes contribuições dos nossos maravilhosos encontros.

À PUC-Rio, pela justiça, pela ajuda financeira e pela excepcional biblioteca.

Ao Dodô, meu cãozinho, fiel companheiro de todas as horas.

À Deus, por todos os momentos vividos e superados nesse processo. 


\section{Resumo}

Silva, Ana Lucia Vaz; Lelis, Isabel; Castro, Mônica Rabello. Números reais no Ensino Médio: identificando e possibilitando imagens conceituais. Rio de Janeiro, 2011. 333p. Tese de Doutorado Departamento de Educação, Pontifícia Universidade Católica do Rio de Janeiro.

O estudo dos números reais com ênfase no aluno do nível médio da escola básica é pouco explorado nas pesquisas em Educação Matemática no Brasil e em outros países. Constatamos, na revisão bibliográfica, que os números racionais e irracionais não fazem parte do repertório de conhecimento de grande parte dos futuros professores, apesar da experiência escolar, como alunos, de vários anos. Trata-se de um campo rico de investigação devido a sua relevância dentro do currículo de Matemática no Ensino Médio. Esta tese analisou as imagens conceituais dos alunos, quando foram estimulados a escrever e/ou falar sobre os números reais, motivados por atividades em que o uso da calculadora foi incentivado. Realizamos uma análise documental desse tema. Elaboramos e aplicamos atividades, priorizando as ideias de densidade e infinito. Utilizamos as representações decimais finitas e infinitas e os números irracionais da forma $\sqrt{n}, \mathrm{n}$ natural, bem como elegemos o algoritmo da divisão como disparador desse processo. A metodologia clínico-qualitativa permitiu-nos coletar os dados em duas etapas: o estudo preliminar exploratório, com a aplicação de um questionário para 70 alunos; e o estudo principal, realizado com 12 alunos, nos quais o aluno foi constantemente solicitado a esclarecer seus julgamentos. $O$ ambiente de pesquisa possibilitou a manifestação e a análise dos julgamentos prototípicos e analíticos que foram identificados por atributos relevantes e irrelevantes. Os resultados apontam para a necessidade de um trabalho efetivo, a partir do algoritmo da divisão, que consolide o conhecimento das representações decimais. $\mathrm{O}$ aumento do repertório de exemplos positivos e negativos relacionados às noções trabalhadas parece estimular descritores de imagem conceitual que são favoráveis para o desenvolvimento do conceito de número real.

\section{Palavras-chave:} clínico.

Número real; imagem conceitual; currículo escolar; Ensino Médio; método 


\section{Abstract}

Silva, Ana Lucia Vaz; Lelis, Isabel (Advisor); Castro, Mônica Rabello. Real numbers in High School: identifying and providing conceptual images. Rio de Janeiro, 2011. 333p. Thesis - Departamento de Educação, Pontifícia Universidade Católica do Rio de Janeiro.

The study of real numbers with an emphasis on the High School students is underexplored in research in Mathematics Education in Brazil and other countries. In the literature review, we note that rational and irrational numbers are not part of the repertoire of knowledge of most prospective teachers, despite their school experience as students, for several years. This is a rich field of research because of its relevance in the Mathematics curriculum in High School. This thesis examined the conceptual images of students after they had been encouraged to write and to talk about real numbers, driven by activities in which the use of a calculator was stimulated. We conducted an analysis of documents of this subject. We elaborated and carried out activities, prioritizing the ideas of density and infinity. We used finite and infinite decimal representations and irrational square roots, and chose the division algorithm as a trigger of this process. The clinical-qualitative methodology allowed us to collect data in two stages: the preliminary exploratory study, by applying a questionnaire to 70 students; and the main study, conducted with 12 students, in which the students were constantly asked to explain their judgments. The research environment has enabled the demonstration and the analysis of prototypical and analytical judgments that were identified by relevant and irrelevant attributes. The results indicate the need for an effective work, from the division algorithm, which consolidates the knowledge about decimal representations. Increasing the repertoire of positive and negative examples related to the notions worked seems to stimulate conceptual image descriptors that are favorable for the development of the concept of real number.

\section{Key-words:}

Real number; conceptual image; school curriculum; High School; clinical method. 


\section{Sumário}

1. Introdução

2. Números reais: o que dizem as pesquisas? ............................. 25

2.1. Pesquisas diagnósticas sobre números reais ........................ 26

2.2. Contribuições do ensino superior ......................................... 32

2.3. Números reais na licenciatura ............................................. 50

2.4. Professores e o ensino de números reais .............................. 60

2.5. Contribuições para esta pesquisa .......................................... 63

3. Construção de conceitos matemáticos: julgamentos prototípicos, analíticos e imagem conceitual ................................ 66

3.1. Construção de conceitos matemáticos .................................... 67

3.2. Matemática elementar e avançada: o caso dos números reais 68

3.3. Imagem conceitual e definição conceitual ................................ 72

3.4. Categorias e protótipos ...................................................... 79

3.4.1. A importância da visualização ......................................... 81

3.4.2. Atributos relevantes e irrelevantes .................................... 82

3.5. Julgamentos prototípicos e analíticos na formação da imagem conceitual

4. Números reais no Ensino Médio …........................................ 86

4.1. O que dizem os documentos curriculares? ........................... 86

4.2. Números reais nos livros didáticos do Ensino Médio ................ 91

4.2.1. Números reais segundo Lima et. al. (2001) ......................... 93

4.2.2. Análise dos livros didáticos de Matemática do Ensino Médio 98

4.3.Como os números reais são apresentados aos futuros

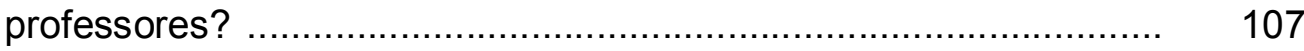

4.3.1. Nos livros de Cálculo ................................................... 108

4.3.2. Nos livros indicados para aprofundamento do tema ............. 110

4.4. Inferências para nossa pesquisa ......................................... 120 


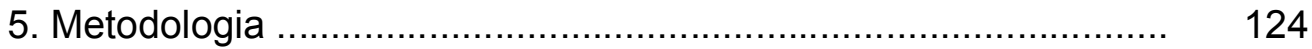

5.1. O estudo preliminar: delimitando caminhos ............................ 126

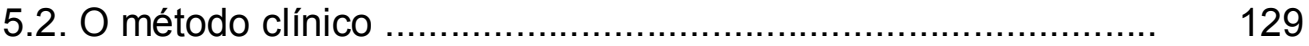

5.3. O estudo principal .......................................................... 132

5.3.1. O desenho da pesquisa ................................................... 133

5.3.2. Os sujeitos do estudo principal .......................................... 134

5.3.3. Instrumentos de coleta de dados ......................................... 137

5.3.3.1. As fichas de atividades ................................................. 138

5.3.3.2. O diário de campo ....................................................... 141

5.3.3.3. As gravações em áudio ................................................ 142

5.3.4. A dinâmica dos encontros ................................................ 143

5.3.5. Procedimentos de análise dos dados ................................ 145

6. Análise das atividades: as imagem conceituais dos alunos ........ 151

6.1. Contexto Inicial ................................................................. 152

6.2. Atividades do Grupo I ......................................................... 154

6.2.1. Aspectos gerais ........................................................... 155

6.2.2. Análises das atividades ................................................... 156

6.2.2.1. Atividade P2: natureza finita e infinita do algoritmo da

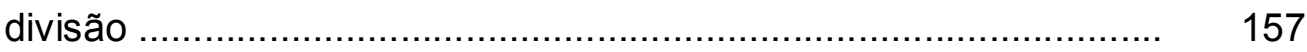

6.2.2.2. Atividade P5: observando números reais na calculadora ... 161

6.2.2.3. Atividade P6: sobre o número irracional 0,121221222... 179

6.2.2.4. Atividade P7: o número $\frac{53}{83}$ e sua representação decimal na calculadora

6.2.2.5. Atividades P8 e P12: dízima periódica e fração geratriz ..... 199

6.2.2.6. Atividade A1: a natureza finita e infinita das representações decimais das frações .......................................... 212

6.2.2.7. Atividades F1, F2 e F3: retomando questões .................... 219

6.2.3. Análise geral do grupo ..................................................... 233

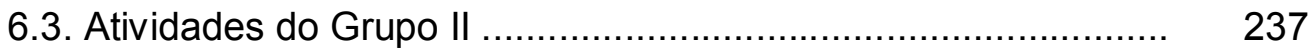

6.3.1 Aspectos gerais ........................................................... 237

6.3.2. Análises das atividades ................................................... 241

6.3.2.1. Atividade P9: os números irracionais da forma $\sqrt{n} \ldots \ldots \ldots \ldots . \quad 241$ 
6.3.2.2. Atividades $\mathrm{P} 13, \mathrm{C} 1$ e $\mathrm{C} 2$ : os números irracionais da forma $\sqrt{n}$ e noções de aproximações racionais e densidade

6.3.2.3. Atividades D3 e F4: operando com os números irracionais da forma $\sqrt{n}$

6.3.2.4. Atividade E4: demonstração da irracionalidade de $\sqrt{2} \ldots \ldots .265$

6.3.2.5. Atividade F1: julgando os números irracionais da forma $\sqrt{n}$

6.3.3. Análise geral do grupo

7. Conclusão

275

8. Referências bibliográficas

288

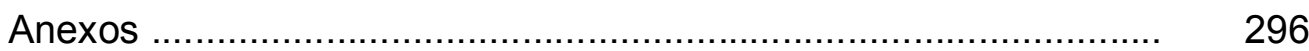




\section{Lista de Figuras}

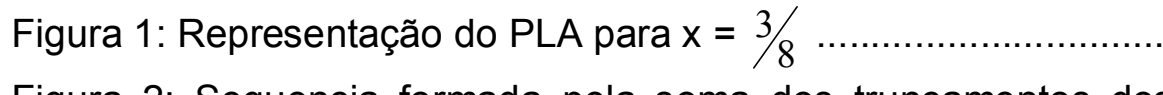

Figura 2: Sequencia formada pela soma dos truncamentos dos números reais $\mathrm{x}$ e $\mathrm{y}$

Figura 3: Determinação do dígito $r_{n}^{x+y}$ no algoritmo vertical da adição (VAA)

Figura 4: Recorte da sessão Flash Matemático da coleção I: sugestão didática para trabalhar com operações de irracionais com o uso de calculadoras

Figura 5: Recorte do manual do professor da coleção III: sugestão 103 didática para trabalhar com radicais com o uso de calculadoras .... 104

Figura 6: Recorte da coleção II: representação geométrica dos números reais na reta

104

Figura 7: Recorte da coleção III sobre o número $\pi \ldots \ldots \ldots \ldots \ldots \ldots \ldots \ldots . . \ldots 106$

Figura 8: Esquema que ilustra o desenho da pesquisa ....

134

Figura 9: Esquema que organiza os dados por grupos de atividades

Figura 10: Atividades do Grupo I ............................................ 147

Figura 11: Atividades do Grupo II ........................................... 148

Figura 12: Esquema didático do grupo I ..................................... 156

Figura 13: Algoritmo relacionado ao item b feito pela dupla BrunoCelso

Figura 14: Algoritmos feitos no quadro pela pesquisadora

Figura 15: Três tentativas de aplicação da regra para obter a fração geratriz

Figura 16: Obtendo a fração geratriz

Figura 17: Quatro exemplos de números decimais e suas correspondentes frações

Figura 18: Resultado matemático sobre decimais finitos 
Figura 19: Algoritmos feitos pela dupla Carla-Isabel .................... 216

Figura 20: Esquema didático do grupo II ................................... 239

Figura 21: Representação geométrica da inequação $|b-N|<\varepsilon . . \quad 240$

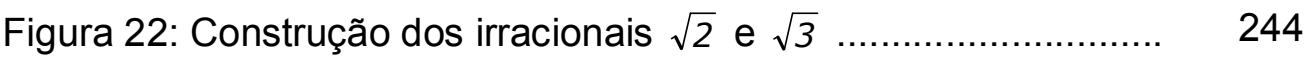

Figura 23: Intervenção da pesquisadora: ideias de erro e aproximação .................................................................. 252

Figura 24: Atividade C2 feita pela dupla Denise - Artur ............... 255

Figura 25: Atividade C2 feita por Carla-Antônio ............................ 256

Figura 26: Atividade C2 feita por Vinícius-Bruno ......................... 256

Figura 27: Atividade C2 feita por Clara-Isabel ............................ 257

Figura 28: Atividade C2 feita por Fernando-Eduardo ................... 258 


\section{Lista de Tabelas}

Tabela 1: Tabulação dos dados obtidos dos participantes sobre o número $0,0555 \ldots$

Tabela 2: Tabulação dos dados obtidos dos participantes sobre o número $34,2727 \ldots$

Tabela 3: Dados selecionados da pesquisa de Igliori e Silva, (1998)

Tabela 4: Duas opções de seqüência de truncamentos para $x=$ $1 / 10$

Tabela 5: Lista das coleções de livros didáticos que analisamos ....

Tabela 6: Sugestões de Lima et al. (2001) para o ensino de números reais

Tabela 7: Respondendo à pergunta 1

Tabela 8: Respondendo à pergunta 2

100

Tabela 9: Respondendo à pergunta 3

102

Tabela 10: Respondendo à pergunta 4

105

Tabela 11: Livros de Cálculo I consultados ...

108

Tabela 12: Os números reais nos livros de Cálculo I

108

Tabela 13: Livros de aprofundamento selecionados para consulta

Tabela 14: Organização e distribuição das atividades nos oito

Tabela 15: Datas e número de participantes de cada encontro .......

Tabela 16: Estudo preliminar da questão P2

157

Tabela 17: Mapa de estudo prévio da questão P5

162

Tabela 18: Atributos de números racionais para os números 1,35353535 e 2,12345678

Tabela 19: Atributos de números irracionais para os números 1,35353535 e 2,12345678

Tabela 20: Exemplos de exercícios que envolvem dízimas periódicas

Tabela 22: Atributos de números racionais ao observar o número $0,121221222 \ldots$

Tabela 23: Atributos de números irracionais ao observar o número $0,121221222 \ldots$

Tabela 25: Atributos de números racionais identificados nas respostas da atividade $\mathrm{P} 7$ 
Tabela 26 Atributos de números irracionais identificados nas

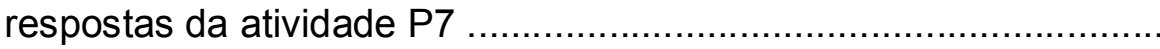
Tabela 27: Estudo prévio da questão P8 ………........................ 200

Tabela 28: Estudo prévio da questão P12 ..................................... 203

Tabela 29: Atividade A1 - $3^{\circ}$ encontro ........................................... 212

Tabela 30: Respostas dos itens b, c e d da atividade A1 ............... 213

Tabela 31: Questão F1 do estudo principal ................................... $\quad 219$

Tabela 32: Respostas obtidas no item a: o número 0,1234. .......... 220

Tabela 33: Respostas obtidas no item b: o número 0,1234. ........... 221

Tabela 34: Respostas obtidas no item c: o número $0,1234 \ldots \ldots \ldots \ldots . \quad 222$

Tabela 35: Respostas obtidas no item d: o número 0,1234... $\underline{n}$

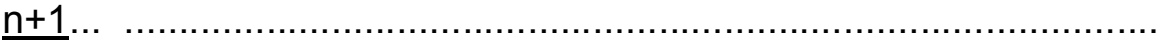

Tabela 36: Respostas obtidas no item g: o número 2,35674126578923.

224

Tabela 37: Questão F2 do estudo principal ................................. 227

Tabela 38: Respostas obtidas na atividade F2 ............................ 228

Tabela 39: Questão F3 do estudo principal

229

Tabela 40: Tabulação das respostas obtidas no item a: sobre a fração $\frac{15}{154}$

Tabela 41: Tabulação das respostas obtidas no item b: sobre a fração $\frac{1387}{80}$.

Tabela 42: Tabulação das respostas obtidas no item c: sobre a fração $\frac{1}{17}$

232

Tabela 43: Estudo prévio da questão P9 ……………................ 242

Tabela 44: Estudo prévio da questão P13 …………..................... 248

Tabela 45: Atividade C1 - $5^{\circ}$ encontro ........................................... 251

Tabela 46: Respostas obtidas na questão C1 ………………….... 252

Tabela 47: Comparando os valores obtidos na atividade para $\sqrt{3}+\sqrt{5}$

Tabela 48: Atividade C2 - $5^{\circ}$ encontro ......................................... 254

Tabela 49: Atividade D3 $-6^{\circ}$ encontro ………………….............. 259

Tabela 50: Atividade E4 $-7^{\circ}$ encontro ……….............................. 266

Tabela 51: Atividade F1 - $8^{\circ}$ encontro ……………………….... 268

Tabela 52: atividade F1 itens e e f $-7^{\circ}$ encontro ……..................... 269 